\title{
ENCRYPTED FUSION OF FACE AND IRIS BIOMETRICS
}

Sivasankari Narasimhan

Assistant Professor, Electronics and Communication Engineering, Mepco Schlenk Engineering College, Virudhunagar Dt, (India).

E-mail: sivani.sivasankari@gmail.com

ORCID: https://orcid.org/0000-0002-3162-4751

Muthukumar Arunachalam

Associate Professor, Electronics and Communication Engineering,

Kalasalingam University, Virudhunagar Dt, (India).

E-mail: Muthuece.eng@gmail.com

ORCID: https:// orcid.org/0000-0001-8070-3475

Recepción: 28/11/2019 Aceptación: 17/03/2021 Publicación: 30/11/2021

\section{Gitación sugerida:}

Narasimhan, S., y Arunachalam, M. (2021). Encrypted fusion of face and iris biometrics. 3C Tecnología. Glosas de innovación aplicadas a la pyme, Edición Especial, (noviembre, 2021), 513-535. https://doi. org/10.17993/3ctecno.2021.specialissue8.513-535 


\section{ABSTRACT}

Security is the main concern in storing databases. Any methods can be used to secure the data. In addition to that identification also done to provide the correct service to the person. In our model we propose the methodology to include biometric fusion and encryption to store and secure user's information. Image fusion is done from the information from different face and iris images. The method of considering only high intensity pixel based image fusion generate new images that hold the attractive information and characteristics of each input image. The fused resulting image is given to the encryption module and encrypted information in stored in database which is further used for identification and authentication. Fusion techniques and lightweight encryption algorithm has been implemented and simulated in MATLAB. The parameters correlation, computation time, Unified averaged changed intensity(UACI), Number of changing pixel rate(NCPR)and Entropy are calculated and the results show the effectiveness of encrypted fusion based on DWT. Entropy has been increased by 67\%. NCPR of 99.6239 and UACI of 3.3004 .

\section{KEYWORDS}

Fusion, Face biometric, Iris biometric, DWT, Encryption. 


\section{INTRODUCTION}

Image fusion is an essential step in multimodal biometrics. Image fusion is a process by which two images are fused together to obtain a single image. Images with different focused regions, images from different modalities or images taken in different times have been fused together to give enhanced results. With change in time, the face images produced by persons may differ due to aging. These images cannot give a clear picture needed for clear identification. Thus, for efficient diagnosis, one needs different multimodal image information in a single image. The features that are not changes more with age is iris. To this point, in research, many fusion techniques based on Iris and face images, were proposed by the researchers. The transform based fusion methods include decomposition of image by Stationary Wavelet Transform (SWT), Discrete Wavelet Transform (DWT), Lifting Wavelet Transform (LWT), Redundancy Discrete Wavelet Transform (RDWT), Dual-Tree Complex Wavelet Transform (DTDWT). These methods have unique drawbacks but all of them share some common drawbacks such as introduced additive noise in fused image.

A multimodal biometric system is developed using fingerprint and iris biometric in Rajbhoj and Mane (2015). The system combines fingerprint and iris at feature level. Single feature vector is obtained by fusing fingerprint and iris image and unique textural pattern from fused image is obtained by efficient wavelet transform. Matching is carried using Hamming distance. Here independent databases are used for face and iris images and each fingerprint is assigned a corresponding iris image.

The multimodal biometric system in ElAlami, Amin, and El-Alfi (2012) combines face and fingerprint biometrics in matching score level. They used the gray-level co-occurrence matrix (GLCM) as an effective method for extracting the texture features in the face recognition and crossing number method is used for fingerprint feature extraction. For matching process they used correlation coefficient as the similarity measure. A multimodal biometric system is developed by combining face and fingerprint biometric by score level fusion. According to $\backslash$ cite $\{19\}$ face recognition is done using PCA and fingerprint recognition is done using minutiae matching and Gabor filtering.

A multimodal biometric system in Krishneswari and Arumugam (2012) combines palm print and fingerprint in feature level. Palm print and finger print images were fused using 
wavelet based image fusion techniques with min-min approximation. Features were extracted using Discrete Cosine Transform (DCT) and feature reduction is done. In their work also independent databases are used for palm print and fingerprint and they are combined by assigning a fingerprint image to each palm print image. Their shows that multi modal biometrics are more efficient than conventional palm print based methods.

Usman et al. (2017) explains about, light weight encryption algorithm which will fit for IoT. The single input image is authenticated using encryption algorithm.

In the proposed method, source images are decomposed into low-level sub band, high-level sub bands using DWT. Next, low-level sub-images are again decomposed into low and high level images. In two level decomposition, iris image is fused.

Our project gives the following significant works

1. Dual modal biometric as face and iris recognition fusion have been taken.

2. Encryption to safeguard the fused database.

3. Analysis of security concerns in both fusion and encryption.

The remaining sections are organized as follows: section 2 provides the proposed methods and section 3 gives the implementation results followed by conclusion in section 4 .

\section{PROPOSED METHODOLOGY}

The proposed DWT consists of four steps: Enhancement, decomposition, fusion and encryption. The block diagram of the proposed DWT-based face iris fusion is shown in Figure 1. Now let us see the process involved and used components in the process one by one. 


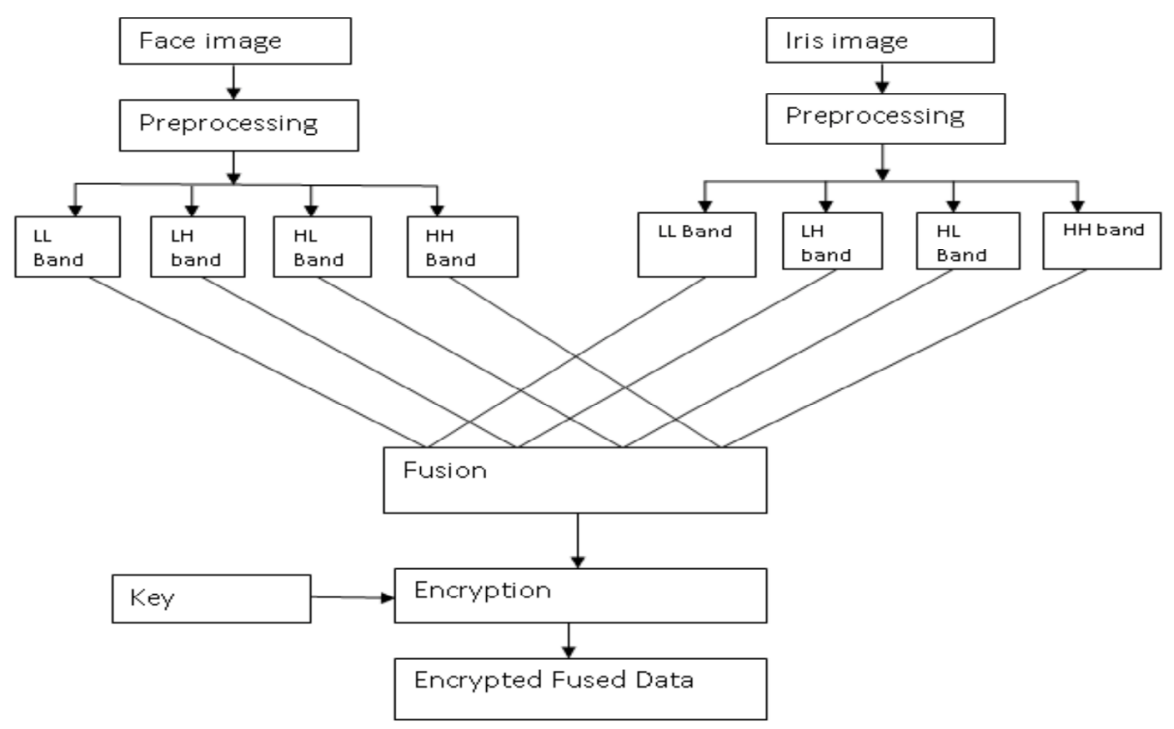

Figure 1. Overall block diagram.

Source: own elaboration.

Step1: Face image and iris images are preprocessed. Color images are converted into gray scale images. They have been resized into 256×256

Step 2: As per Discrete Wavelet Transform (DWT), approximation, horizontal, vertical and diagonal details have been found out for two levels for both images. The individual sub images are compared and the highest intensity value image is taken for fused image.

Step 3: The image is encrypted using secure light weight encryption algorithm.

\subsection{DWT ALGORITHM}

The Haar wavelet illustrates the desirable properties of wavelets in general. It can generally use for image denoising. It uses the orthonormal basis vector in the rows of $\left[\begin{array}{cccc}1 & 1 & 1 & 1 \\ 1 & 1 & -1 & -1 \\ 1 & -1 & 0 & 0 \\ 0 & 0 & 1 & -1\end{array}\right]$. DWT demonstrates the localization: the first row $(1,1,1,1)$ term gives the average signal value; Second row $(1,1,-1,-1)$ places the signal in the left side of the domain; and $(1,-1,0,0)$ places it at the left of the left side. The explanation of DWT is 
available in "Discrete wavelet transform" (2008). First, the samples are passed through a low pass filter with impulse response $g(n)$, Hence the convolution operation between input image function and impulse function has been taken place.

$$
y(n)=\sum_{k=0}^{n-1} x(k) \cdot g(n-k)
$$

The signal is decomposed simultaneously using high pass filter (h). So, the two filters are related to each other and they are known as a quadrature mirror filter.

$$
\begin{aligned}
& y_{\text {low }}(n)=\sum_{k=-\infty}^{\infty} x(k) g(2 n-k) \\
& y_{\text {high }}(n)=\sum_{k=-\infty}^{\infty} x(k) h(2 n-k)
\end{aligned}
$$

With the sub sampling factor 2, the process is continued and all the levels are cascaded. For the representation of images in multi resolution wavelets can be used. If it has to be converted into other domain, the properties in one domain are easily separable and scalable. There is a unique set of expansion coefficients in every representable function of Fourier kernals.

\subsection{FUSION}

The performance of biometric system is improved by choosing correct fusion. In our approach feature level fusion is used. The feature vectors at second level of DWT are combined to produce the fused image. For verification of individual persons biometric, different images of any biometric can be fused. But authentication purpose, two different biometrics have been connected.

\subsection{ENCRYPTION}

Various encryption algorithms are being developed still now. For message encryption Symmetric encryption algorithm is used. In our algorithm, due to space complexities, light weight encryption algorithm is used. Usually, encryption operation is performed with specified number of bits known as key. With a 64 bit initial key further rounds of keys are generated. Key expansion is done as per the logic provided by Usman et al. (2017). The initial key is set as "AAAAAAAAAAAA" for this algorithm. It can be expanded for next 
rounds. The key expansion diagram is shown in Figure 2. The confusion and diffusion are introduced by ' $\mathrm{F}$ ' function in it.

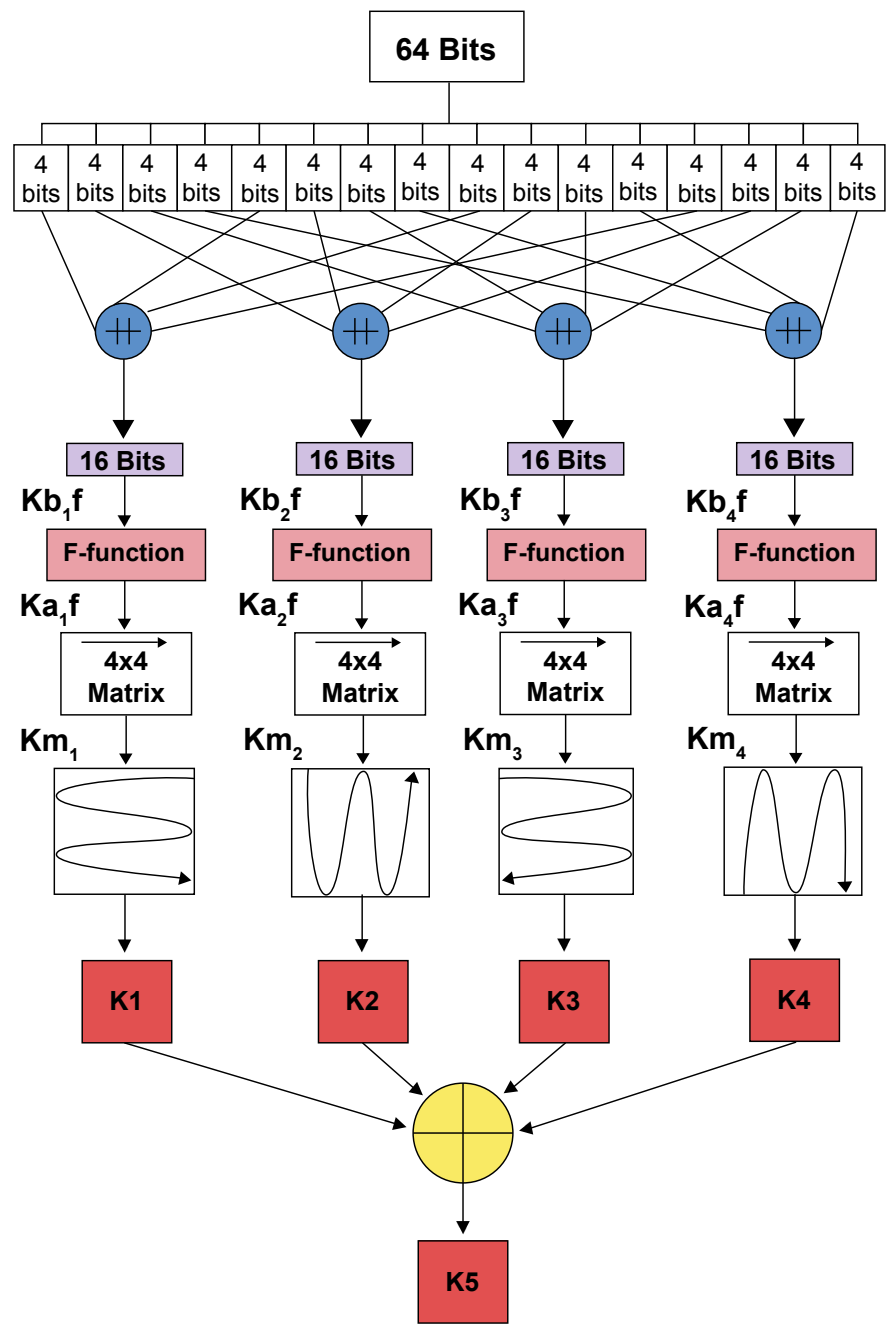

Figure 2. Key expansion.

Source: (Usman et al., (2017).

As per the figure, from the initial key $\mathrm{K} 5$ is derived as $\mathrm{XOR}$ function of $\mathrm{K}_{1}, \mathrm{~K}_{2}, \mathrm{~K}_{3}, \mathrm{~K}_{4}$. The function ' $F$ ' has done some permutations which gets 16 bits input and 16 bit output. Then encryption process is done as per equation 6 . The intermediate ciphertext is given as:

$$
I C_{i, j}=\left\{\begin{array}{c}
P x_{i, j} \Theta K_{i} \\
P x_{i, j+1 \oplus E f} \\
P x i, j-1 \oplus E f
\end{array} ;\right.
$$


$\mathrm{IC}_{\mathrm{i}, \mathrm{j}}$ is the intermediate result. $\mathrm{Px}_{\mathrm{i}, \mathrm{j}}$ is the plaintext. If $\mathrm{j}=1$ or 4 first equation is performed. If $\mathrm{j}=2$ second operation is performed. If $\mathrm{j}=3$ third operation is done. The final encryption operation is performed as the concatenation result of fifth round.

\section{C=IC51 || IC52 || IC53 || IC54}

The encrypted information is stored in database, further it is used for identification.

\section{IMPLEMENTATION RESULTS}

Yale database is taken for face image in our experiment, which contains 15 subjects each of which have 15 images under different postures. The size of the image is $320 \times 243$. CASIA database is taken for iris images which contains 36 iris subjects and 7 for each. For our experiments, first 7 images of face are merged with first 15 images of IRIS database.

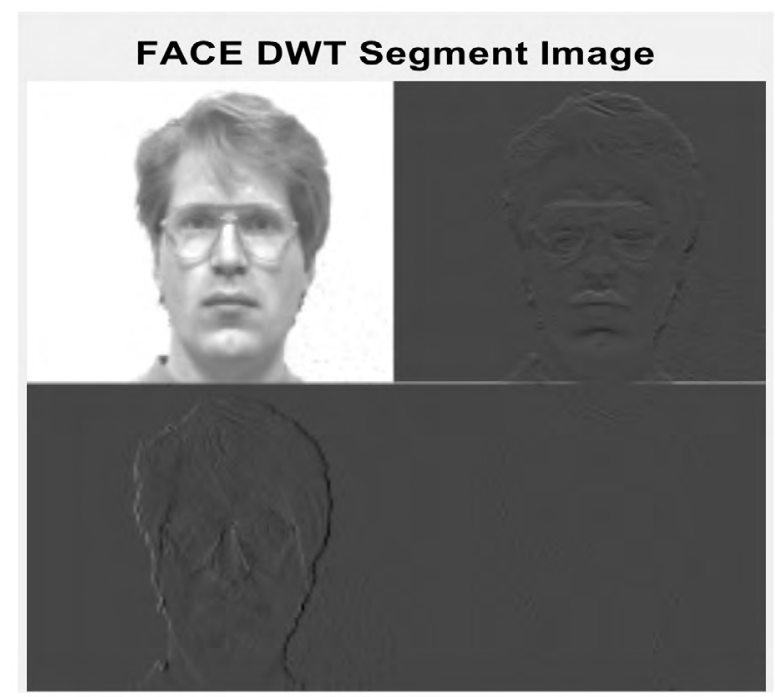

Figure 3. FACE DWT segment image.

Source: own elaboration.

First both face and iris images are undergone for DWT segmentation. The segmented image is shown in Figures 3 and 4. 


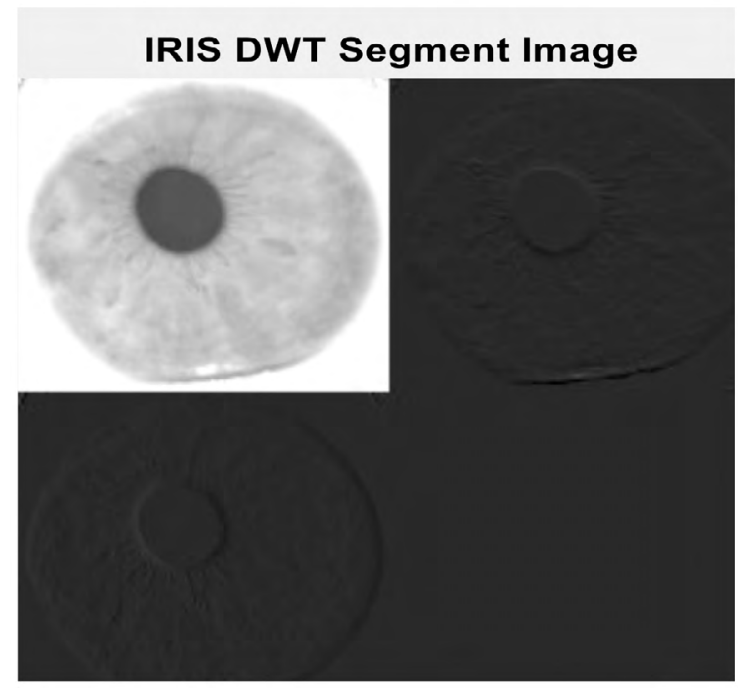

Figure 4. IRIS DWT segment image.

Source: own elaboration.

The images are getting fused by highest intensity feature level fusion logic. The fused image is shown in Figure 5.

\section{Fusion Image of iris \& FACE}

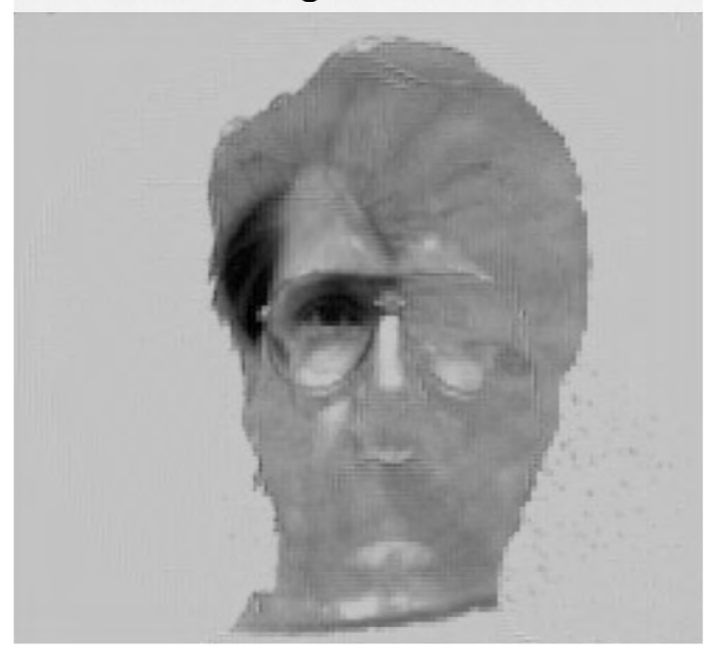

Figure 5. Fused image.

Source: own elaboration.

But fused image is intermediate stage. It is not visible by anybody. This image is going for encryption. In our algorithm, we are using light weight encryption algorithm. Hence it 
can be used for chip implementation. The fused image is resized to 256x256 image. After encryption we got the image as given in Figure 6.

\section{Original}

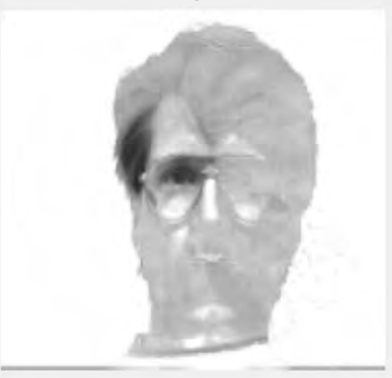

Encrypted

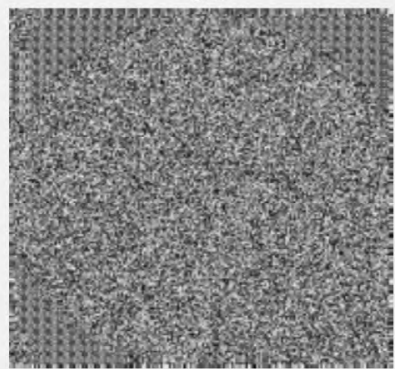

\section{Decrypted}

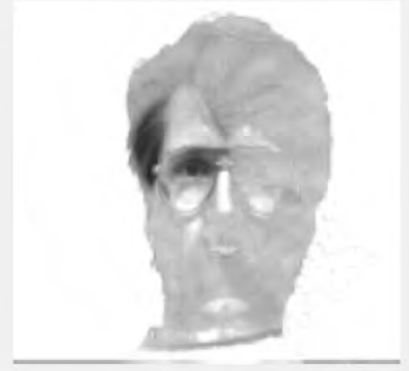

Figure 6. Encryption and Decryption by correct key.

Source: own elaboration.

When the same user is used for authentication the correlation score should be high. The encrypted image and original image are shown with their histograms in Figure 7.

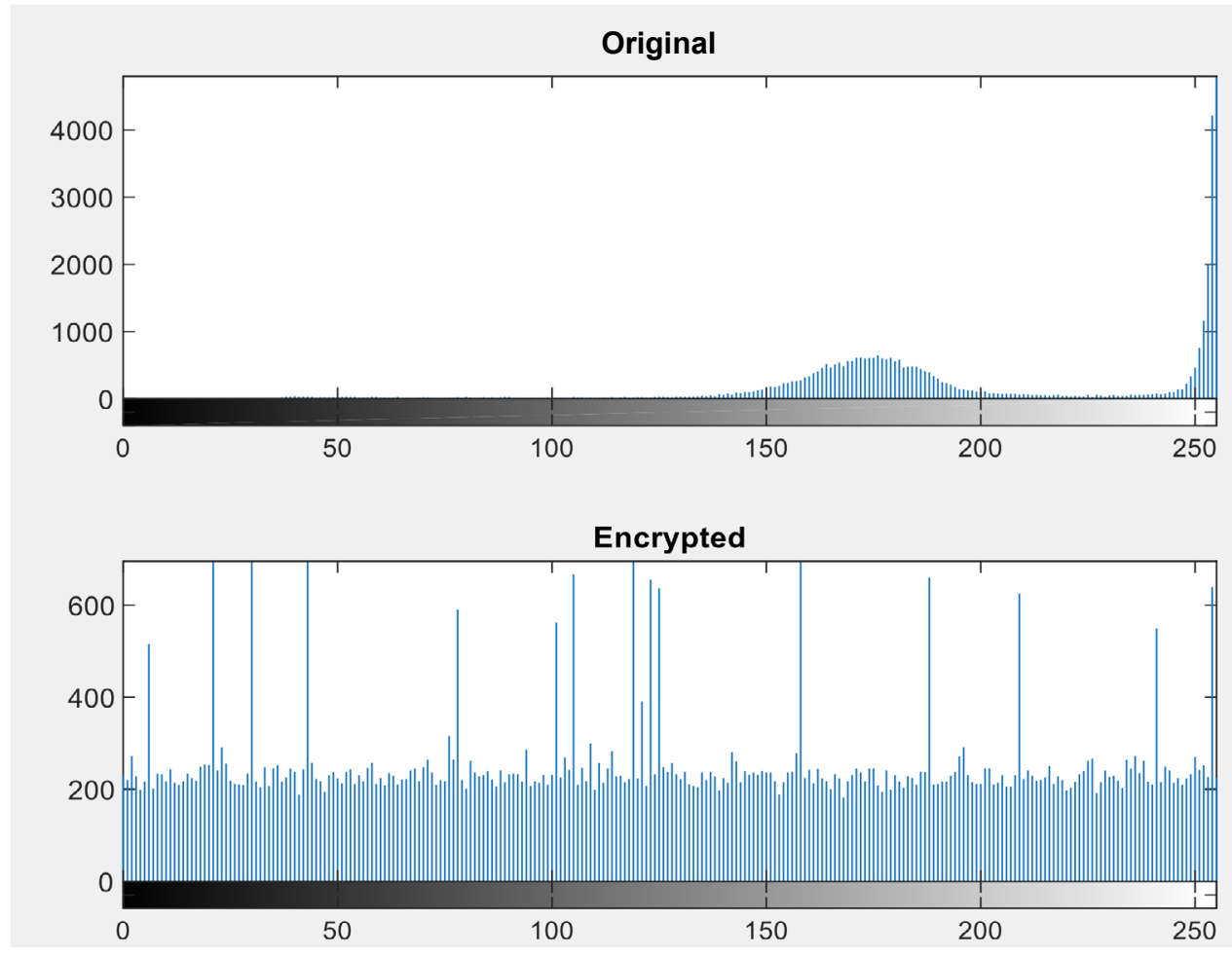

Figure 7. Correlation of original and encrypted image.

Source: own elaboration. 
Now, the image correlation and entropies have been calculated. The scatter plot of image is given in Figure 8.
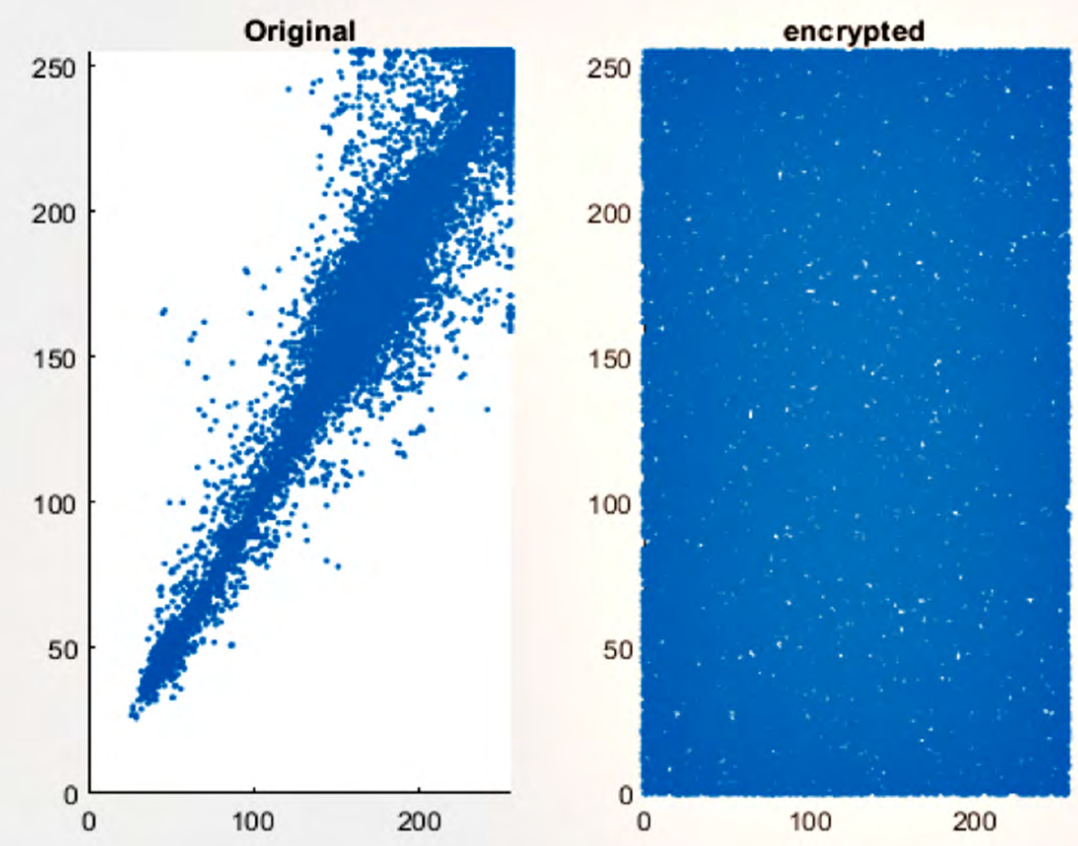

Figure 8. Scatter plot of encrypted image.

Source: own elaboration.

\subsection{EVALUATION PARAMETERS}

Image Entropy: the encryption algorithm adds extra information to the data so as to make it difficult for the intruder to differentiate between the original information and the encrypted information. Entropy is defined as:

$$
\mathrm{H}(\mathrm{I})=\sum_{i=1}^{2^{8}} P\left(I_{i}\right) \log _{b} P\left(I_{i}\right)
$$

How much entropy is higher, more in formations are present, and hackers cannot find it easily. The results for the 35 image fusion are given in Table 1. Average of 7.7512 for the encrypted image is obtained. Almost $69 \%$ of entropy is increased, compared with original fused image. 
Correlation: dependency of statistical relationship between two images is said to be correlation. A well-designed cipher should not possess any relationship with original message.

In our experiment the correlation coefficient is calculated for original fused message and encrypted images. The correlation coefficient is defined as:

$$
\gamma_{x, y}=\frac{\operatorname{cov}(x, y)}{\sqrt{D(x), D(y)}}
$$

where $\operatorname{cov}(\mathrm{x}, \mathrm{y}), \mathrm{D}(\mathrm{x})$ and $\mathrm{D}(\mathrm{y})$ are covariance and variances of variable $\mathrm{x}$ and $\mathrm{y}$ directions respectively. For ideal cipher case $\gamma$ should be equal to 0 and for the worst case $\gamma$ will be equal to 1 .

In our experiment the original fused image has correlation coefficient of 0.98157 , whereas the encrypted images have -0.109 correlation coefficient. (They are negatively correlated).

Number of changing pixel rate (NGPR): this is the parameter for testing the encryption against differential attacks. As per Wu, Noonan and Agaian (2011) the range of percentage is [0-100]. When it is zero it remains that all the pixels are having the same value. Glearly NCPR concentrates on absolute number of pixels which changes values in differential attacks.

$$
N P C R: N\left(C^{1}, C^{2}\right)=\sum_{i, j} \frac{D(i, j)}{T} x 100 \%
$$

Where $\mathrm{C}^{1}, \mathrm{C}^{2}$ are the before and after one pixel image in cipher.

$$
D(i, j)=\left\{\begin{array}{l}
0, \text { if } C^{1}(i, j)=C^{2}(i, j) \\
1, \text { if } C^{1}(i, j) \neq C^{2}(i, j)
\end{array}\right\}
$$

$\mathrm{T}$ denotes the total number of pixels in ciphertext. The average value we obtain for NCPR is 99.6239 .

\section{Unified averaged changed intensity (UAGI):}

UACI concentrates on the averaged difference between two paired ciphertext images.

$$
U A C I: U\left(C^{1}, C^{2}\right)=\sum_{i, j} \frac{a b s\left(C^{1}(i, j)-C^{2}(i, j)\right)}{F . T} x 100 \%
$$


Where $\mathrm{F}$ is the maximum supported pixel value compatible with the cipher text. In our experiment $\mathrm{F}$ is taken as 255. The average value we obtained from this as 3.3004

Table 1. Analysis of parameters with key 'AAAAAAAAAAAAAAAA'.

\begin{tabular}{|c|c|c|c|c|c|c|c|c|}
\hline \multirow[b]{2}{*}{ Image 1} & \multirow[b]{2}{*}{ Image 2} & \multicolumn{2}{|c|}{ Correlation } & \multirow{2}{*}{$\begin{array}{c}\text { Total } \\
\text { Encryption } \\
\text { time (ms) }\end{array}$} & \multirow[b]{2}{*}{$\begin{array}{c}\text { NCPR } \\
(\%)\end{array}$} & \multirow[b]{2}{*}{ UACI (\%) } & \multicolumn{2}{|c|}{ Entropy } \\
\hline & & $\begin{array}{l}\text { Original } \\
\text { Image }\end{array}$ & $\begin{array}{l}\text { Encrypted } \\
\text { Image }\end{array}$ & & & & $\begin{array}{c}\text { Original } \\
\text { image }\end{array}$ & $\begin{array}{c}\text { Encrypted } \\
\text { image }\end{array}$ \\
\hline \multirow{6}{*}{$\begin{array}{c}\text { Subject } 1 . \\
\text { glasses }\end{array}$} & Iris 1 & 0.9782 & -0.0206 & 9.172 & 99.661 & 3.082 & 4.124 & 7.9318 \\
\hline & Iris $1 b$ & 0.9751 & -0.0246 & 8.113 & 99.653 & 2.2138 & 4.2116 & 7.9311 \\
\hline & Iris $1 \mathrm{c}$ & 0.9848 & -0.0048 & 8.096 & 99.597 & 3.8024 & 4.2159 & 7.9152 \\
\hline & Iris 1d & 0.9689 & -0.0138 & 8.165 & 99.676 & 1.6850 & 4.2351 & 7.9251 \\
\hline & Iris $1 \mathrm{e}$ & 0.9831 & -0.0138 & 8.093 & 99.679 & 3.4871 & 4.2117 & 7.9319 \\
\hline & Iris $1 f$ & 0.9800 & -0.0168 & 8.453 & 99.557 & 2.4720 & 4.3174 & 7.9716 \\
\hline \multirow{6}{*}{$\begin{array}{c}\text { Subject } 2 . \\
\text { glasses }\end{array}$} & Iris 2 & 0.9831 & -0.0058 & 8.003 & 99.565 & 3.8076 & 4.2263 & 7.2146 \\
\hline & Iris $2 b$ & 0.9817 & -0.0014 & 8.158 & 99.623 & 3.7036 & 4.2247 & 7.2308 \\
\hline & Iris 2c & 0.9737 & -0.0096 & 8.034 & 99.586 & 1.8903 & 4.2126 & 7.2310 \\
\hline & Iris $2 d$ & 0.9737 & -0.0096 & 9.002 & 99.586 & 1.8903 & 4.2329 & 7.5151 \\
\hline & Iris $2 e$ & 0.9845 & -0.0077 & 8.197 & 99.627 & 4.0569 & 4.2352 & 7.4151 \\
\hline & Iris $2 f$ & 0.9795 & -0.0021 & 8.232 & 99.632 & 2.6016 & 4.1247 & 7.5309 \\
\hline \multirow{7}{*}{$\begin{array}{l}\text { Subject } 3 . \\
\text { glasses }\end{array}$} & Iris 3 & 0.9821 & -0.0139 & 8.358 & 99.632 & 2.6081 & 4.2147 & 7.9308 \\
\hline & Iris $3 b$ & 0.9856 & -0.0071 & 8.264 & 99.577 & 3.1991 & 4.2146 & 7.9310 \\
\hline & Iris $3 c$ & 0.9854 & -0.0142 & 8.875 & 99.636 & 3.1645 & 4.2359 & 7.9151 \\
\hline & Iris $3 d$ & 0.9854 & -0.0142 & 8.270 & 99.636 & 3.1645 & 4.2359 & 7.9151 \\
\hline & Iris $3 e$ & 0.9798 & -0.0100 & 8.522 & 99.630 & 2.4692 & 4.2147 & 7.9309 \\
\hline & Iris $3 f$ & 0.9889 & -0.0115 & 8.415 & 99.638 & 4.5532 & 4.3374 & 7.9726 \\
\hline & Iris $3 g$ & 0.9880 & -.0169 & 8.679 & 99.676 & 3.9941 & 4.2663 & 7.9146 \\
\hline \multirow{7}{*}{$\begin{array}{c}\text { Subject4. } \\
\text { glasses }\end{array}$} & Iris 4 & 0.9804 & -0.0124 & 8.508 & 99.600 & 3.3139 & 5.1418 & 7.9561 \\
\hline & Iris $4 b$ & 0.9804 & -.0167 & 8.426 & 99.638 & 3.1778 & 5.0205 & 7.9314 \\
\hline & Iris 4c & 0.9804 & -.0167 & 8.426 & 99.638 & 3.1778 & 5.0205 & 7.9314 \\
\hline & Iris 4d & 0.9804 & -0.0167 & 8.8108 & 99.638 & 3.1778 & 5.0205 & 7.9314 \\
\hline & Iris $4 \mathrm{e}$ & 0.9795 & -0.0102 & 8.8440 & 99.658 & 3.8139 & 5.2303 & 7.9743 \\
\hline & Iris $4 f$ & 0.9808 & -0.0152 & 9.0458 & 99.627 & 4.0870 & 5.2835 & 7.9536 \\
\hline & Iris $4 \mathrm{~g}$ & 0.9801 & -0.0167 & 9.1555 & 99.615 & 4.1067 & 5.2935 & 7.9506 \\
\hline \multirow{7}{*}{$\begin{array}{l}\text { Subject05. } \\
\text { glasses }\end{array}$} & Iris 5 & 0.9822 & -0.0037 & 9.0337 & 99.624 & 3.5343 & 5.0542 & 5.0542 \\
\hline & Iris $5 b$ & 0.9848 & -0.0039 & 9.0955 & 99.551 & 4.0488 & 5.0956 & 7.9943 \\
\hline & Iris $5 c$ & 0.9833 & -0.0091 & 9.1250 & 99.644 & 3.4544 & 4.8371 & 7.9641 \\
\hline & Iris $5 d$ & 0.9833 & -0.0091 & 9.0839 & 99.644 & 3.4544 & 4.8371 & 7.9641 \\
\hline & Iris $5 \mathrm{e}$ & 0.9837 & -0.0050 & 8.8077 & 99.679 & 3.4289 & 4.7934 & 7.9699 \\
\hline & Iris $5 f$ & 0.9855 & -0.0030 & 9.1667 & 99.575 & 4.1457 & 5.1360 & 7.9884 \\
\hline & Iris $5 g$ & 0.9855 & -0.0030 & 8.8251 & 99.575 & 4.1457 & 5.1360 & 7.9884 \\
\hline
\end{tabular}

Source: own elaboration. 
This work has been accomplished with first image of yale database and CASIA database of second image. Various parameters have analyzed in this methodology and the parameters are given in Table 1.

Key sensitivity: all encryption algorithms have to be designed in such a way that, if one bit is changed almost $60 \%$ of the data should be changed in ciphertext. The changes in seed key value for the same biometric subjects are taken and the result is compared in Table 2. The distribution of histogram is given in Table 3 .

Table 2. Comparison of various keys to show the key sensitivity.

\begin{tabular}{|c|c|c|c|c|c|c|c|}
\hline Image & Image 2 & Key & $\begin{array}{c}\text { Correlation of } \\
\text { Original fused } \\
\text { Image }\end{array}$ & $\begin{array}{c}\text { Correlation } \\
\text { of Encrypted } \\
\text { Image }\end{array}$ & $\begin{array}{c}\text { Total } \\
\text { Encryption time } \\
\text { (ms) }\end{array}$ & ((NCPR) & (UACI) \\
\hline $\begin{array}{c}\text { Subject 1 } \\
\text { glasses }\end{array}$ & Iris 1 & $\begin{array}{c}\text { AAAAAAAA } \\
\text { AAAAAAAA }\end{array}$ & 0.9782 & -0.0206 & 9.172423 & 99.66 & 3.082 \\
\hline $\begin{array}{c}\text { Subject 1 } \\
\text { glasses }\end{array}$ & Iris 1 & $\begin{array}{c}\text { ABACADAE } \\
\text { AFAA1234 }\end{array}$ & 0.9796 & -0.0134 & 10.920902 & 99.6363 & 3.1735 \\
\hline $\begin{array}{c}\text { Subject 3 } \\
\text { glasses }\end{array}$ & Iris 3 & $\begin{array}{c}\text { ABACADA } \\
\text { EAFAA1234 }\end{array}$ & 0.0110 & 0.9867 & 8.282109 & 99.6323 & 3.4111 \\
\hline
\end{tabular}

Source: own elaboration.

Table 3. Comparison of different correlation histograms and scatter diagrams.

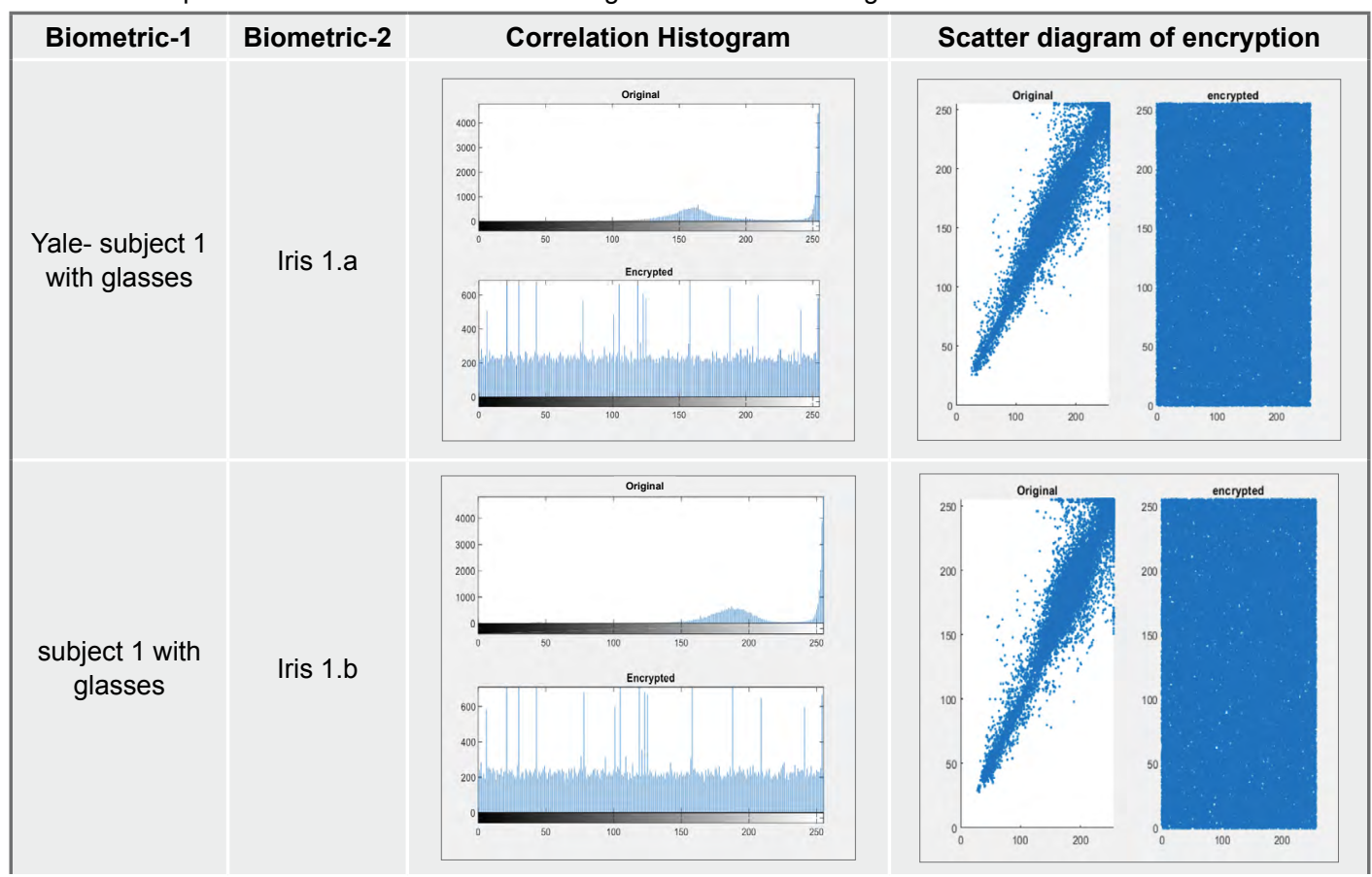




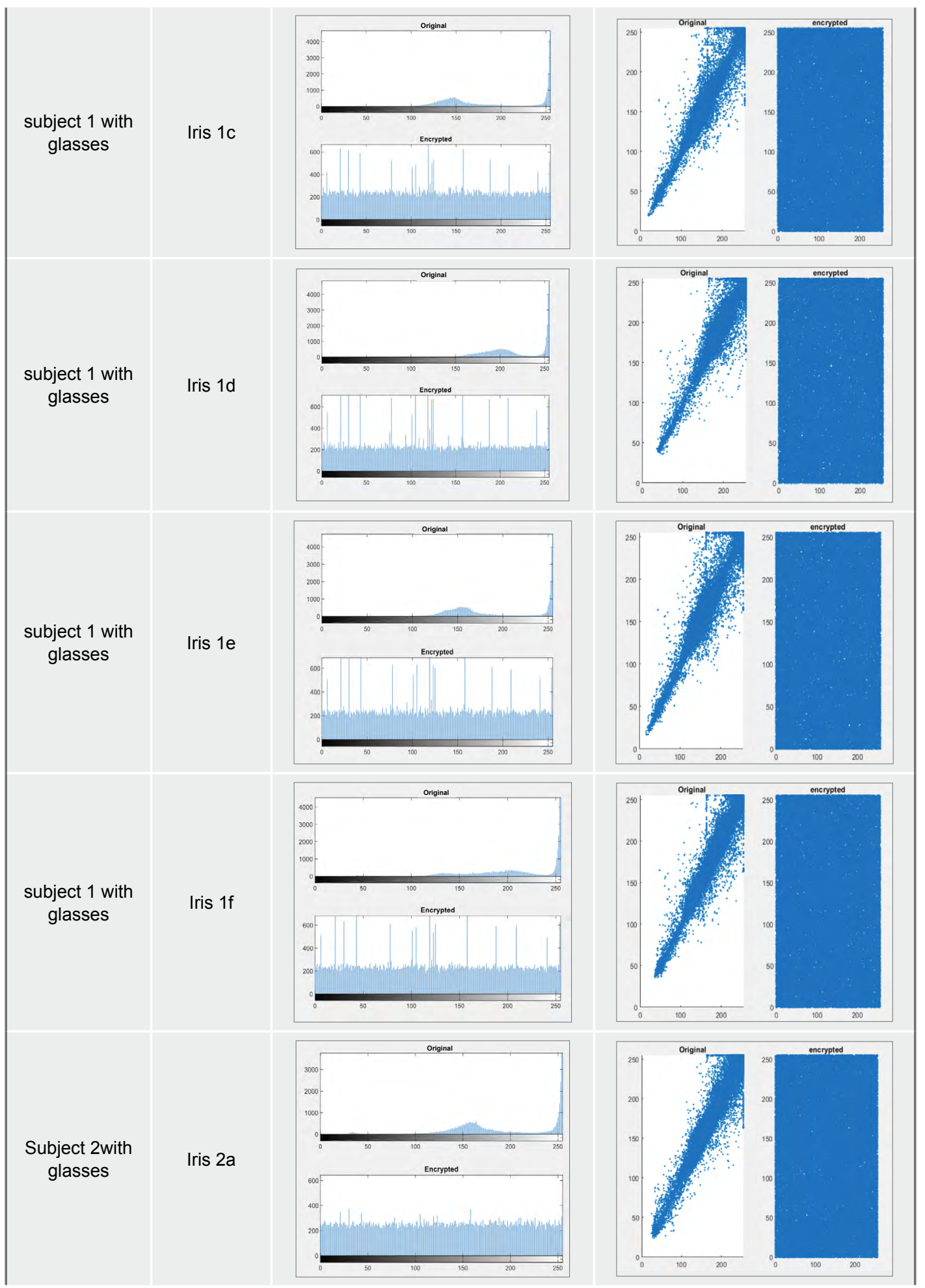




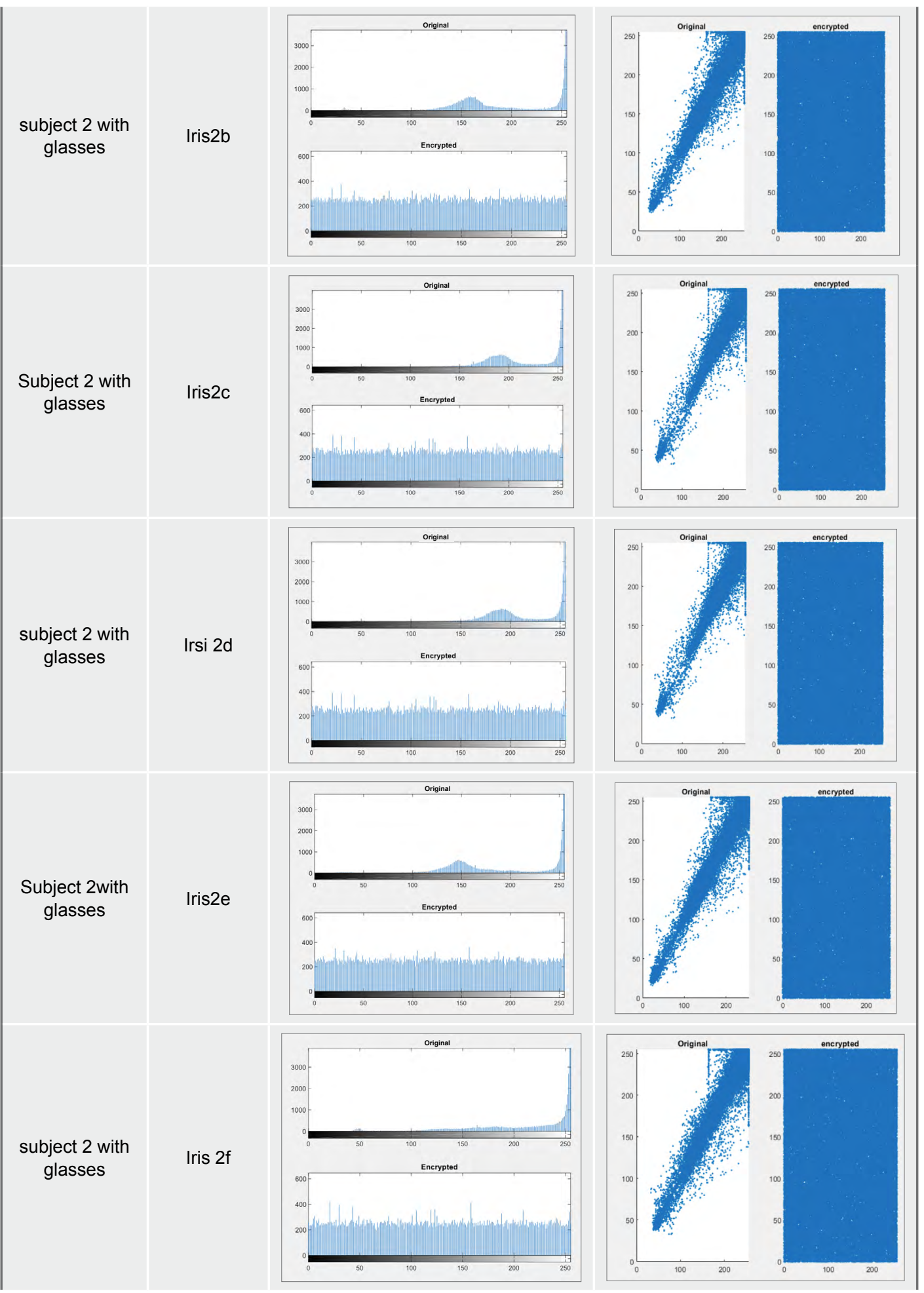




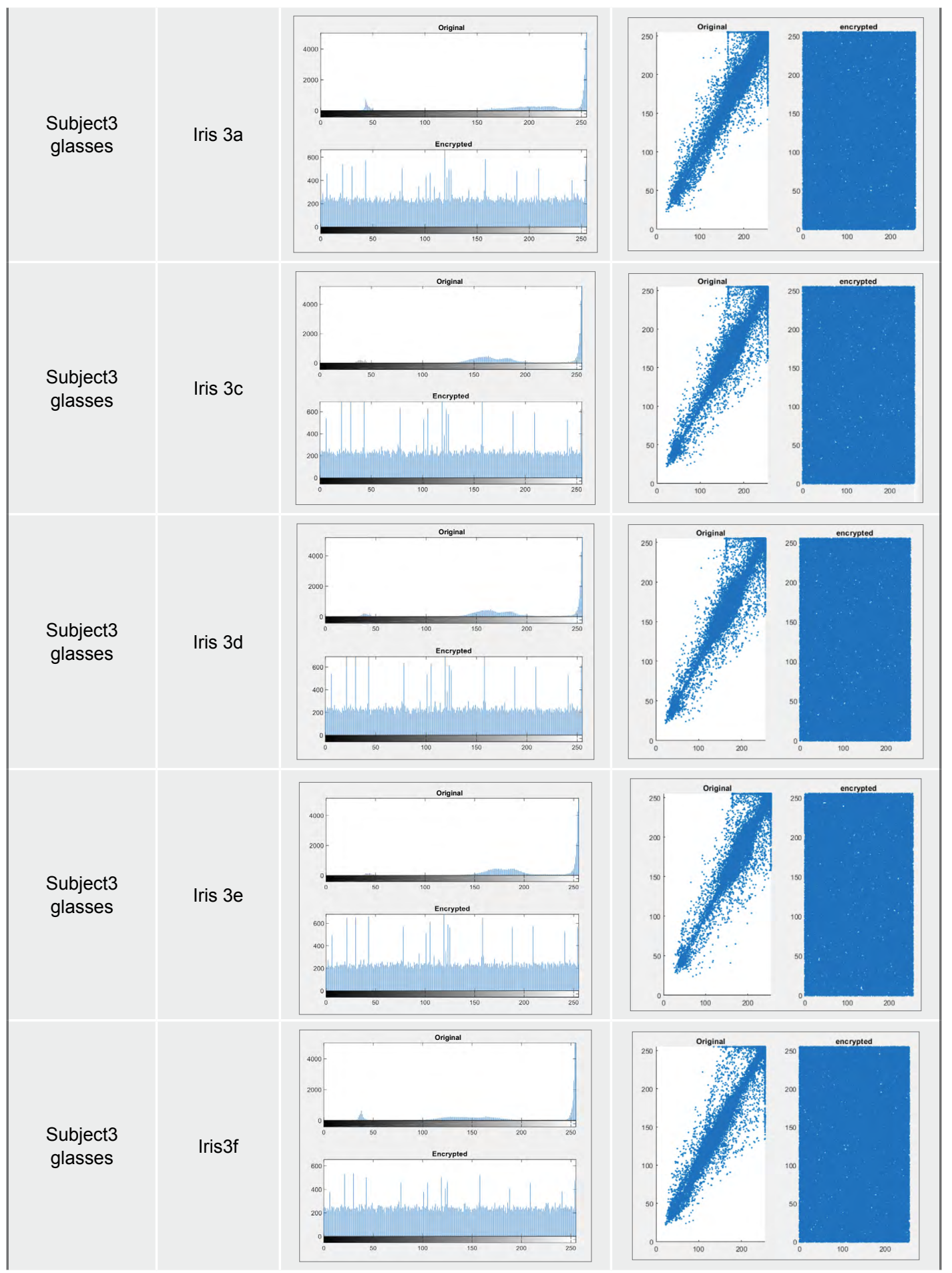




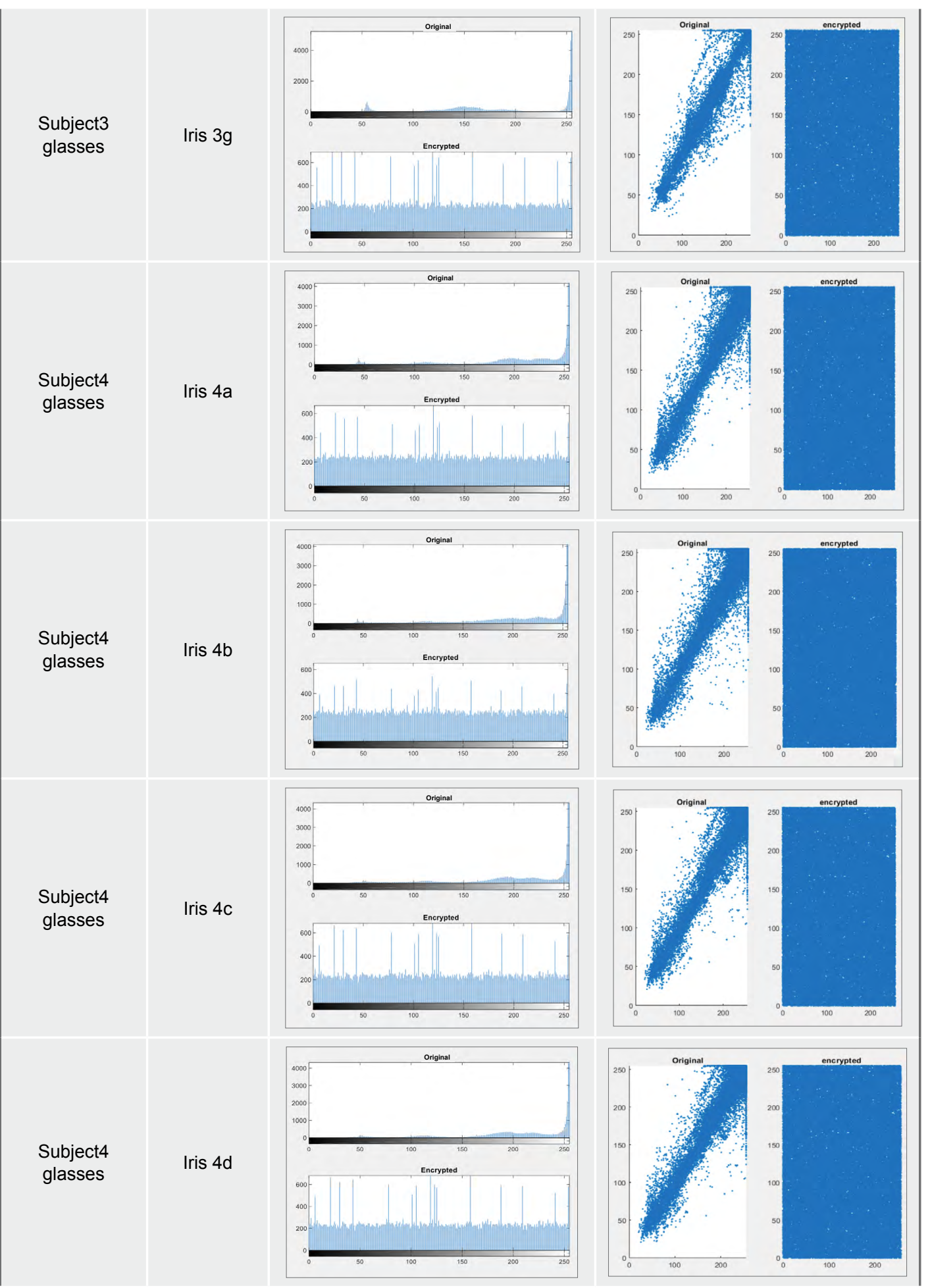




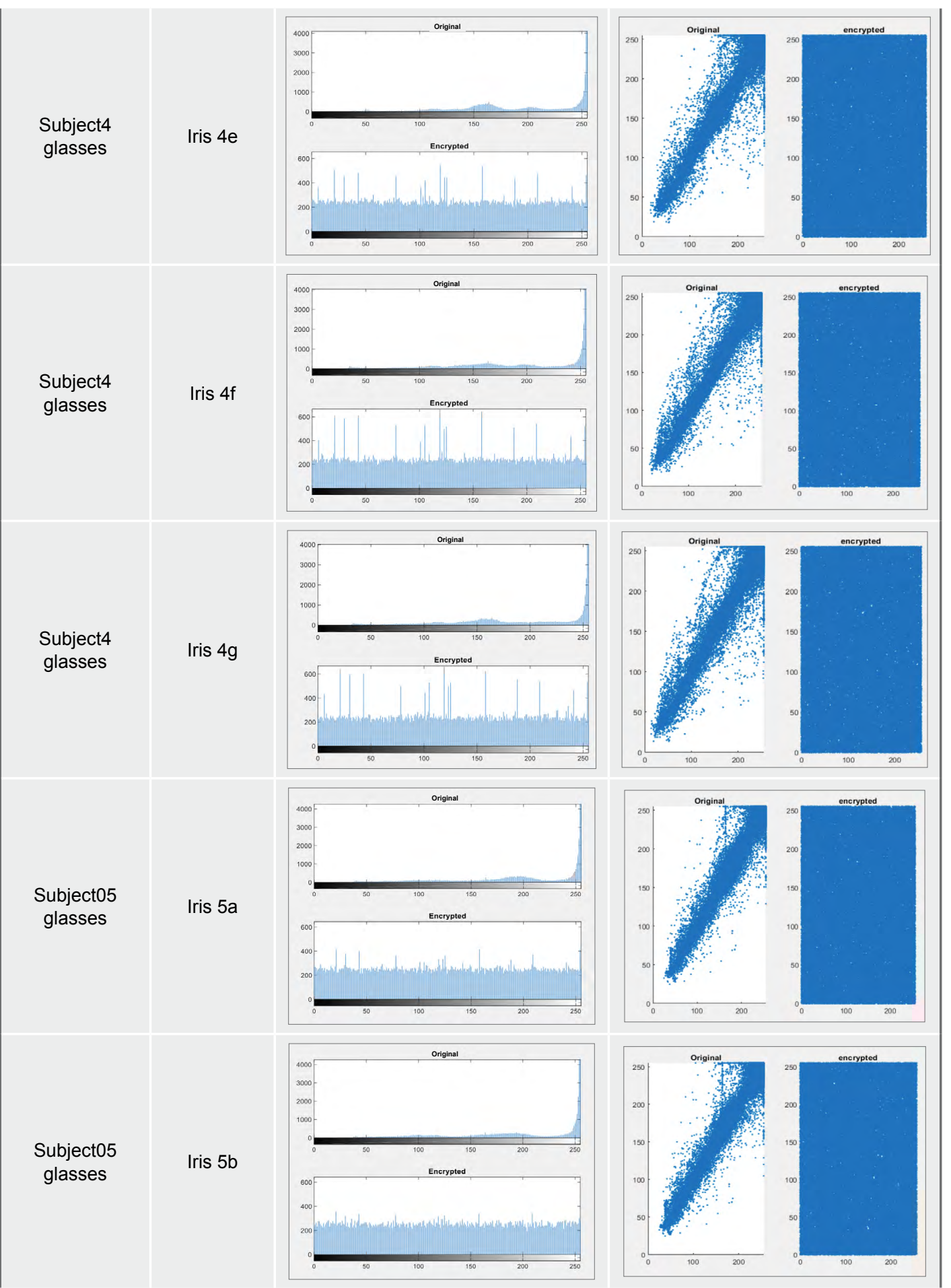




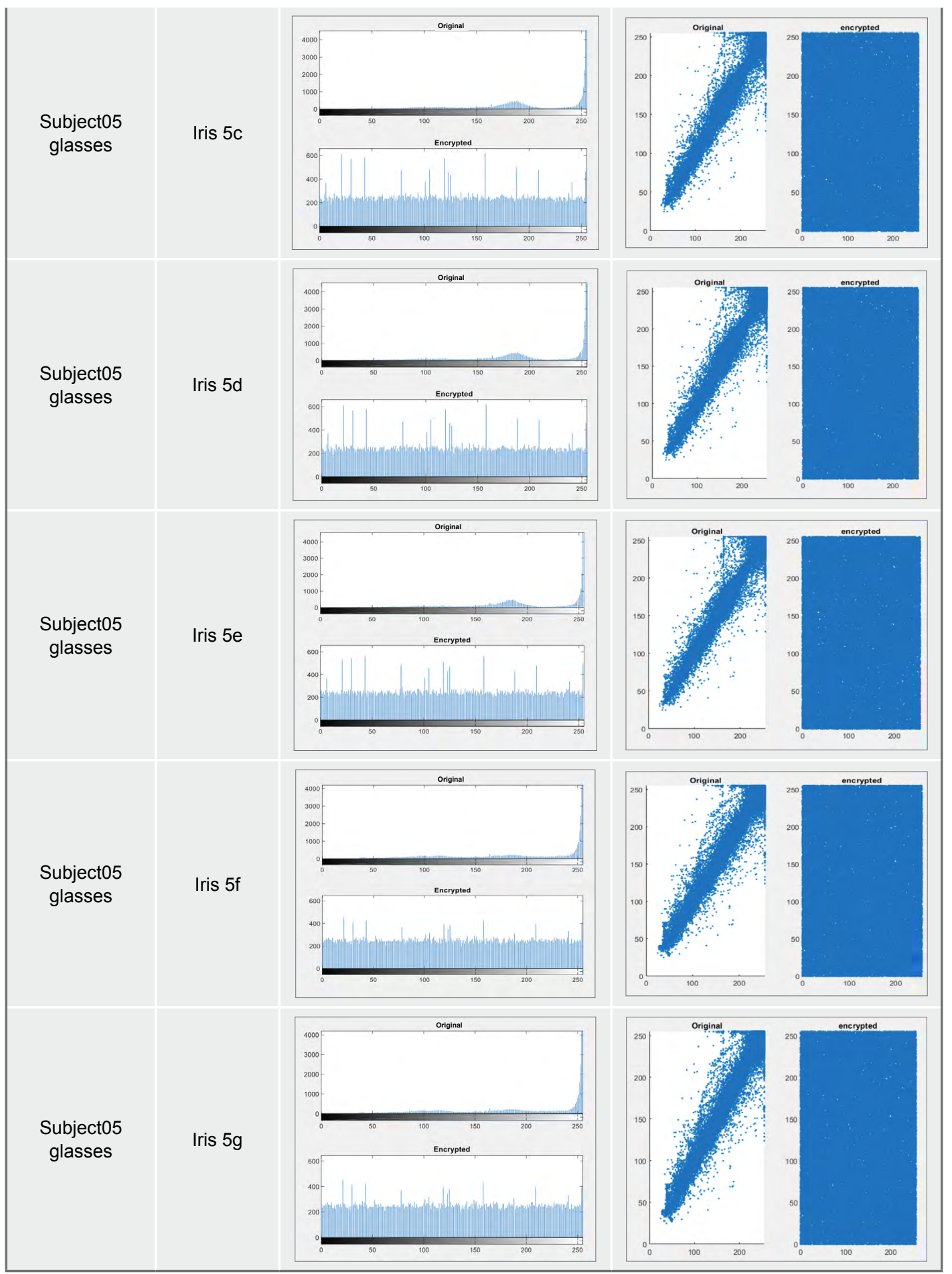

Source: own elaboration. 


\section{CONCLUSIONS}

In this algorithm, a feature level fusion method to fuse the two biometrics is introduced. Encryption algorithm to do the operations in a simple manner without the need of S-box is performed for fused image. Finally, the security parameters such as entropy, correlation, computation time, Unified averaged changed intensity, Number of changing pixel rate are calculated. As a future scope, this logic can be implemented with some public key algorithms to implement in ATM.

\section{REFERENCES}

Discrete wavelet transform. (2008). https://en.m.wikipedia.org/wiki/Discrete_ wavelet_transform

ElAlami, M. E., Amin, A. E., \& El-Alfi, A. E. (2012). A Personal Identification Framework based on Facial Image and Fingerprint Fusion Biometric. International Journal of Computer Applications, 51(7), 41-48. https://doi.org/10.5120/8058-1411

Krishneswari, K., \& Arumugam, S. (2012). Multimodal Biometrics using Feature Fusion. Fournal of Computer Science, Science Publications, 8(3), 431-435. https://doi. org/10.3844/jcssp. 2012.431 .435

Rajbhoj, S. M., \& Mane, P. B. (2015). An Approach of Combining Iris and Fingerprint Biometric At Image Level in Multimodal Biometrics System. International fournal of Soft Computing and Engineering, 5(1), 102-106. https://docplayer.net/151961568An-approach-of-combining-iris-and-fingerprint-biometric-at-image-level-inmultimodal-biometrics-system.html

Usman, M., Ahmed, I., Aslam, M. I., Khan, S., \& Shah, U. A. (2017). SIT: A Lightweight Encryption Algorithm for Secure Internet of Things International fournal of Advanced Computer Science and Applications, 8(1), 1-10. https://doi.org/10.14569/ IJACSA.2017.080151

Wu, Y., Noonan,J. P., \& Agaian, S. (201 1). NPCR and UACI Randomness Tests for Image Encryption. Cyber Journals: Multidisciplinary Journals in Science and Technology, 
Journal of Selected Areas in Telecommunications (FSAT), 31-38. https://citeseerx.ist.psu. edu/viewdoc/download?doi=10.1.1.390.2127\&rep=rep $1 \&$ type =pdf 
